\title{
Testing of the SOLPS-ITER code at Globus-M2 spherical tokamak with detached divertor
}

\author{
Daria Sorokina, ${ }^{1, *}$, Ilya Senichenkov ${ }^{1}$, Elena Vekshina $^{1}$, and Vladimir Rozhansky ${ }^{1}$ \\ ${ }^{1}$ Peter the Great Saint Petersburg Polytechnic University, Polytechnicheskaya, 29, 195251, Saint \\ Petersburg, Russia
}

\begin{abstract}
In according to a present understanding of Scrape-Off Layer (SOL) physics, future thermonuclear devices like ITER, DEMO and beyond, require high radiation regimes in order to reduce heat loads on tokamak divertor. Recent experiments at ASDEX Upgrade, JET and other tokamaks demonstrated that such regimes might be achieved by the seeding of the radiative impurities. In the present paper the modeling of the high radiation regimes and the transition to the detachment at the Globus-M2 spherical tokamak is performed by the SOLPS-ITER transport code. The obtained modeling results for GLobus-M2 tokamak demonstrate the trend similar to what is observed at larger machines, e.g. AUG and JET. The significant reduction of peak power density at the outer target plate and transition to the detachment with High Field Side High Density (HFSHD) formation at the inner plate was achieved with impurity seeding rate almost equal to the deuterium puff (in el/sec). However, unlike AUG, further increasing of the seeding rate leads not to a formation of the radiative X-point, but to a radiative collapse. This is caused by smaller machine size, which allows the impurity neutrals to penetrate easier into the confined region. It was noticed that starting with attached divertor the inner target transits to the detachment earlier than the outer one.
\end{abstract}

\section{Introduction}

Both future fusion devices and steady state tokamak based neutron sources require reduced energy flux densities at the divertor in order to decrease the plasma facing surfaces damage $[1,2]$. According to the experimental [3] and analytical [4, 5] results the SOL width is significantly narrow, so power crossing the separatrix affects only a thin band at the target plates. A promising solution to the power exhaust challenge is the detachment [6], when energy dissipates due to the radiation and ionization losses. Such regime can be achieved by the density rise or by the injection of seed impurity.

High radiation scenarios with detached divertor targets were observed at AUG [9-11] and JET $[12,13]$ with different seeded gases such as krypton, nitrogen, and neon. While $\mathrm{Kr}$ increases the radiated power in the core and unfavorably reduces the core confinement, both $\mathrm{N}$ and $\mathrm{Ne}$ seems to efficiently increase the radiation in the SOL and can be used to achieve

*Corresponding author: daria.s.sorokina@gmail.com 
the detachment $[14,15]$. $\mathrm{N}$ seems to retain and radiate in the divertor region and slightly affect the core confinement. However, $\mathrm{N}$ can react with hydrogen isotopes with a formation of compounds unfavorable for a thermonuclear reactor operation [16]. Ne is a noble gas, but for tested devices parameters neon seems to transport significantly through the separatrix, and like $\mathrm{Kr}$, cause the strong radiation in the core, which leads the transition to a low confinement or a radiative collapse [15]. Therefore, although the impurity choice is still discussed for large-scale future machines [17], for the smaller ones the confinement reduction caused by Ne seeding is crucial [15].

The analysis of the transition to the detachment at the compact spherical tokamaks can improve understanding of the impurity dynamics in the device volume, and complement the results achieved at the conventional tokamaks. Furthermore, the fusion neutron source, the key element of the fusion-fission hybrid reactor, consider to be based on spherical tokamak [2]. Hybrid reactor demands the steady state conditions, so the device seems to need the surface thermal protection like the detachment.

In the present paper the problem of the transition to the detachment is studied through numerical modeling at Globus-M2 spherical tokamak, which is the upgraded Globus-M machine [18].

\section{Methods}

The Globus-M2 is a small spherical tokamak $(\mathrm{R}=36 \mathrm{~cm} . \mathrm{a}=24 \mathrm{~cm})$, which is the upgraded Globus-M machine. Globus-M2 has former vacuum chamber, but the magnetic coil system has been upgraded resulting in increase of toroidal field and plasma current by a factor of 2.5 , thus reaching values $\mathrm{B}=1.0 \mathrm{~T}$ and $\mathrm{I}=500 \mathrm{kA}$ correspondingly. That allows increasing of energy, power of the NBI auxiliary heating [18], discharge power, plasma temperature and energy confinement time as compared to Globus-M.

The Globus-M2 has not reached the planned parameters yet, and there are no experimental data with high magnetic field and high current, therefore any prediction may be done only through the numerical modeling. In this publication the modeling of $2 \mathrm{D}$ transport in the plasma edge was performed by SOLPS-ITER transport code [19, 20], which is based on the B2 plasma solver and includes the self-consistent model for drifts, plasma currents and electric potential. The code is widely used on many machines like ITER [21], AUG [22], JET [23], MAST [24], Alcator-Cmod [25], Globus-M [26].

The flux surface shapes to create the SOLPS-ITER computational mesh based on the $2 \mathrm{D}$ plasma equilibrium, and in present modeling the equilibrium of the shot $\# 34410(B=0,4 \mathrm{~T}$. $\mathrm{I}_{\mathrm{p}}=198 \mathrm{kA}$ ) from the Globus-M database was chosen (the poloidal and toroidal magnetic fields increased by 2.5 times) in order to match after multiplying by the factor of 2.5 the expected parameters of the upgraded device. The created structured quadrangular orthogonal mesh (Fig. 1) covers the area going from the boundary inside the confined region to the line of first tangency with the material wall of the chamber. 


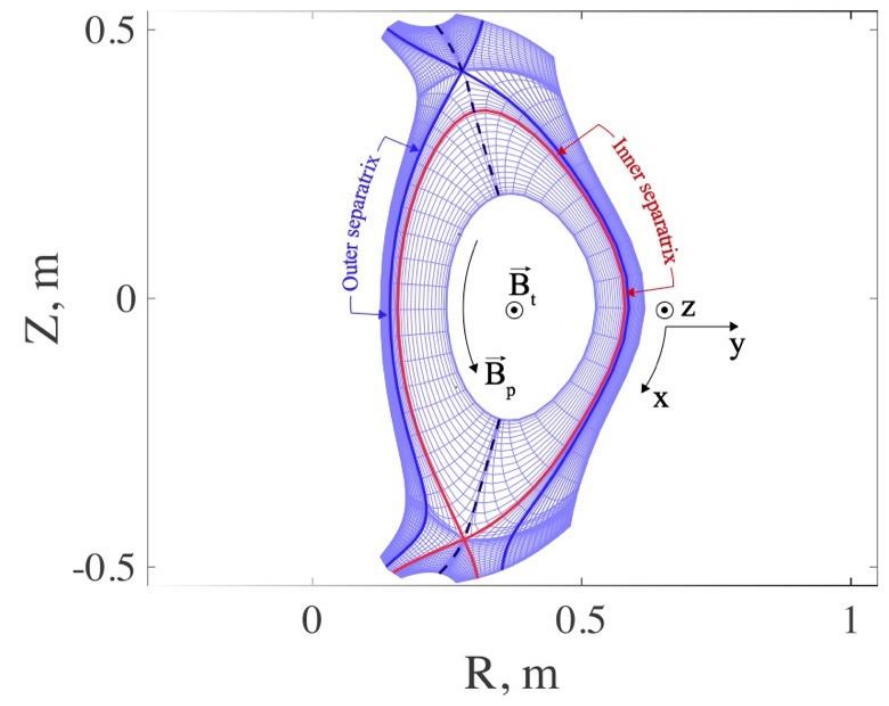

Fig. 1. Computational mesh for the SOLPS-ITER code

So far it is impossible to determine the anomalous transport coefficients in the edge plasma with sufficient accuracy, and in the SOLPS-ITER code the coefficients are the external input parameters, which appear in the radial particle and energy flux densities expressions in the form:

$$
\begin{gathered}
q=-\chi n \nabla_{\perp} T+\ldots \\
\Gamma=V n-D \nabla_{\perp} n+\cdots
\end{gathered}
$$

$\chi$ - the anomalous thermal conductivity, $V$ - pinch velocity, $D$ - the anomalous diffusion.

In present modeling these coefficients (Fig. 2) are chosen the same as those used in the modeling of the Globus-M shot \#34410 [26], except the ion heat conductivity (the anomalous contribution is introduced).

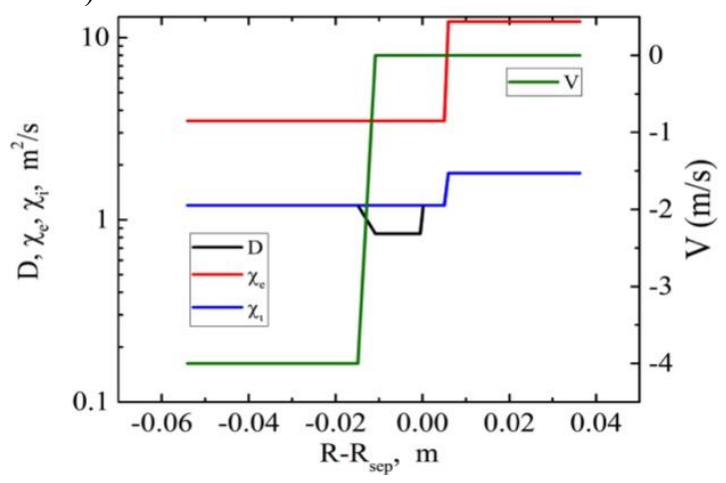

Fig. 2. Anomalous transport coefficients in the equatorial midplane, zero corresponds to the separatrix position (here and below) 


\section{Results and Discussion}

\subsection{Results of the operating modes modeling of Globus-M2 spherical tokamak without seed impurities}

In this section the results of modeling of three Globus-M2 regimes with parameters, predicted though the ASTRA code modeling in [18], are presented. The electron density and the absorbed discharge power are $3.8 \cdot 10^{19} \mathrm{~m}^{-3}$ and $0.6 \mathrm{MW}$ for the first regime, $9.0 \cdot 10^{19} \mathrm{~m}^{-3}$ and 1.3 MW for the second regime, and $3.8 \cdot 10^{19} \mathrm{~m}^{-3}$ and $1.3 \mathrm{MW}$ for the third one. The values are set at the inner boundary of the computational domain (here and below). Calculated profiles of energy flux densities at the divertor are presented in Fig. 3. For the low power case and the high-density case the values are not crucial. For the regime with big power and low-density the values exceed the material limit of $10 \mathrm{MW} / \mathrm{m}^{2}$.

For low-density cases both inner and outer targets remain attached, and in this sense there is target symmetry. With the density increasing in the big power case the inner target transits to the detachment earlier than the outer one, which corresponds to an asymmetry, and then both targets becomes detached. Therefore, the transition to the detachment was achieved in the second case due to the density rise.
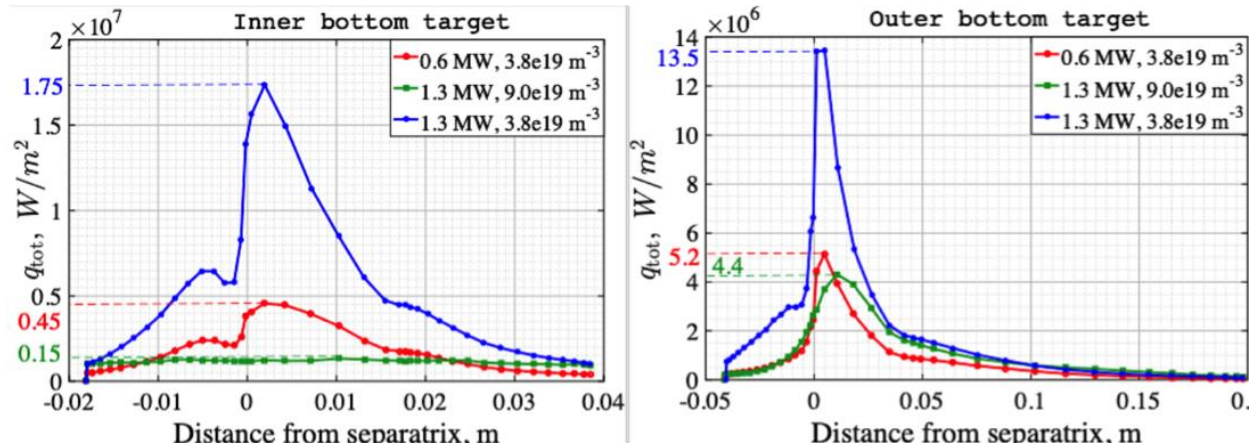

Fig. 3. Energy flux densities at the inner target (left) and at the outer target (right) for three cases

\subsection{Results of the detached divertor mode modeling of Globus-M2 spherical tokamak with nitrogen seeding}

In accordance with the small size of the studied device the nitrogen was chosen as a seeded gas. The transition to the detachment was modeled for two discussed above operating scenarios with same electron density equal $3.8 \cdot 10^{19} \mathrm{~m}^{-3}$ and differing about two times power, 0.6 MW and 1.3 MW.

For the big power regime the $\mathrm{N}$ seeding rate varies from $1.0 \cdot 10^{18}$ to $5.0 \cdot 10^{19}$ atoms $/ \mathrm{s}$. The detachment occurs at a value of $5.0 \cdot 10^{19}$ atoms/s, and it is a threshold, and further slight increase in seeding intensity leads to a radiative collapse. The difference in the regimes with attached and detached divertor is illustrated by the $2 \mathrm{D}$ plots of main plasma parameters for the pure deuterium case and case with the intensive nitrogen puff with rate $5.0 \cdot 10^{19}$ atoms $/ \mathrm{s}$, which are plotted at Fig. 4-6.

With the increase of the seeding rate the electron density growth and the temperatures drop are obtained. With the rate equal $5.0 \cdot 10^{19}$ atoms/s the strong density growth is achieved at the inner divertor target while at the outer target there is a density decrease, which corresponds the formation of the HFSHD - the region with high electron density at the divertor target in the high field side, and the same distribution for the radiating impurity, see Fig. 4. Simultaneously, electron and ion temperatures reduction is achieved at both targets, 
presented in Fig 5. With the seeding rate $5.0 \cdot 10^{19}$ atoms/s the temperature drops to $1 \mathrm{eV}$ at the inner target, where three-body recombination and ion-neutral collisions become efficient, and particles start to recombine in the divertor volume. As a result, the energy fluxes from the core do not reach the divertor, but dissipate by radiation and ionization above the targets, which is presented in Fig. 6. Also, it is worth noting the significant nitrogen transport into the core which cause the temperature decrease and radiation losses into the confined region.

The result of transition to the detachment is a significant reduction of the energy fluxes densities at the divertor, which is presented in Fig. 7. For the big power regime the decrease of the peak values from $17.5 \mathrm{MW} / \mathrm{m}^{2}$ to $0.1 \mathrm{MW} / \mathrm{m}^{2}$ at the inner target and from $13.5 \mathrm{MW} / \mathrm{m}^{2}$ to $5 \mathrm{MW} / \mathrm{m}^{2}$ was achieved. The similar reduction of energy flux may be achieved by density rise from 3.8 to $9.0 \cdot 10^{19} \mathrm{~m}^{-3}$ (Fig. 3).

For low power regime the seeding rate varies from $2.6 \cdot 10^{17}$ to $1.6 \cdot 10^{19}$ atoms $/ \mathrm{s}$. The obtained results are similar to the ones presented above, however the detachment at the inner target can be achieved with a wider range of the injection rates $-4-16 \cdot 10^{18}$ atoms $/ \mathrm{s}$.

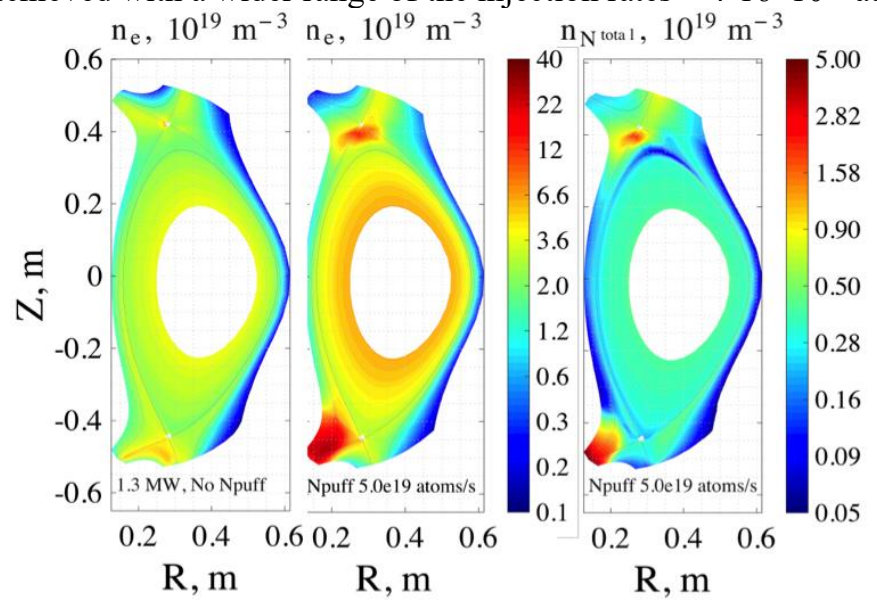

Fig. 4. 2D plots of electron density for regime without impurity and regime with seeding rate $5.0 \cdot 10^{19}$ at/s (left) and 2D plot of impurity density (right).
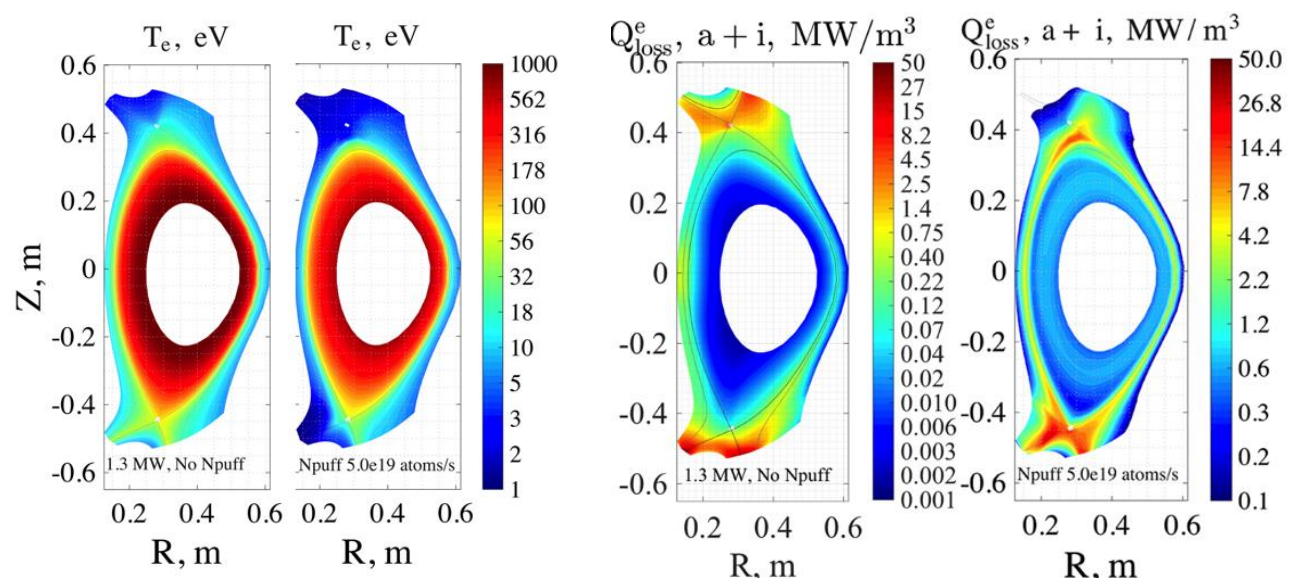

Fig. 5. 2D plots of electron temperature for Fig. 6. 2D plots of losses due to ionization and regime without impurity and regime with radiation for regime without impurity and regime seeding rate $5.0 \cdot 10^{19}$ atoms $/ \mathrm{s}$.

with seeding rate $5.0 \cdot 10^{19}$ atoms/s. 

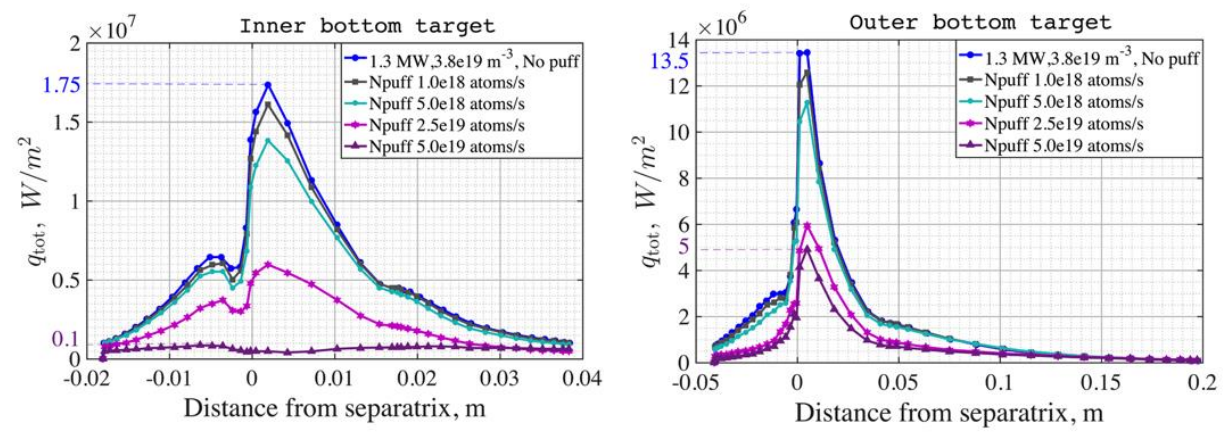

Fig. 7. Energy fluxes densities along the inner divertor target (left) and the outer divertor target (right) for regimes with different impurity seeding rates

\section{Conclusions}

In the present paper the 2D transport modeling of three operational modes of Globus-M2 tokamak is performed by the SOLPS-ITER transport code. For the first time the transition to the detached divertor in the compact spherical tokamak is studied. The results do not contradict to experimental and analytic data from other devices and contribute to the understanding of the detachment at devices with a small aspect ratio.

For Globus-M2 tokamak the detachment at both divertor targets and the formation of radiating X-point is not achieved, as the further increase of the seeding rate leads to the radiative collapse. It occurs at the values of the seeding rate almost equal to the fueling rate, if measured in el/s. The exact value depends on discharge power. At this threshold value the energy flux density reduces 2-4 times at the outer target. The inner target fully detaches, and the energy flux reduces by more than order of magnitude. Thus, the existence of an operation window with significant reduction of target energy flux and without crucial damage to the confinement is demonstrated for compact spherical tokamak Globus-M2.

The obtained results can be used for the experiment on obtaining the detachment in the study of divertor protection methods at the Globus-M2 [27].

The work is done in the Peter the Great Saint Petersburg Polytechnic University under support of the Russian Scientific Foundation, Grant No. 17-12-01020.

\section{References}

1. R.A. Pitts et al., J. Nucl. Mater. 415, S957 (2011)

2. B.V. Kuteev et al., Nucl. Fusion 55, 073035 (2015)

3. T. Eich et al., Nucl. Fusion 53, 093031 (2013)

4. R.J. Goldston, Nucl. Fusion 52, 013009 (2012)

5. V.A. Rozhansky et al., Plasma Phys. Contr. Fusion 60, 035001 (2018)

6. S. Krasheninnikov and A. Kukushkin, J. Plasma Phys. 83, 155830501 (2017)

7. A.W. Leonard, Plasma Phys. Control. Fusion 60, 044001 (2018)

8. J. Boedo et al., Plasma Phys. Control. Fusion 60, 044008 (2018)

9. S. Potzel et al., Nucl. Fusion 54, 013001 (2014)

10. F. Reimold et al., Nucl. Fusion 55, 033004 (2015) 
11. A. Kallenbach et al., Nucl. Fusion 55, 053026 (2015)

12. A.R. Field et al., Plasma Phys. Control. Fusion 59, 095003 (2017)

13. C. Guillemaut et al., Plasma Phys. Control. Fusion 59, 045001 (2017)

14. M.L. Reinke et al., J. Nucl. Mater. 415, S340-S344 (2011)

15. M. Bernert et al., Nuclear Materials and Energy 12, 111-118 (2017)

16. C. Giroud et al., Nucl. Fusion 53, 113025 (2013)

17. A. Khan et al., in 23rd International Conference on Plasma Surface Interactions in Controlled Fusion Devices (PSI) (2018)

18. V.B. Minaev et al., Nucl. Fusion 57, 066047 (2017)

19. S. Wiesen et al., J. Nucl. Mater. 463, 480-484 (2015)

20. X. Bonnin et al., Plasma and Fusion Research 11, 1403102 (2016)

21. A.S. Kukushkin et al., Fusion Engineering and Design 86, 2865-2873 (2011)

22. R. Schneider et al., Contrib. Plasma Phys. 46, No. 1-2, 3 - 191 (2006)

23. L. Aho-Mantila et al., Plasma Phys. Control. Fusion 59, 035003 (2017)

24. V. Rozhansky et al., Nucl. Fusion 51, 083009 (2011)

25. W.Dekeyser et al., Nuclear Materials and Energy 12, 899-907 (2017)

26. E. Vekshina et al., Plasma Phys. Control. Fusion 58, 085007 (2016)

27. V.B. Minaev et al., Phys.: Conf. Ser. 1094, 012001 (2018) 